\title{
The word-superiority effect: Is its locus visual-spatial or verbal?
}

\author{
LESTER E. KRUEGER \\ The Ohio State University, Columbus, Ohio 43210
}

\begin{abstract}
Hemispheric specialization was used to study letter detection. Better detection of letters in words than nonwords was found only in the right hemifield, both for horizontal (Experiment I) and vertical arrays (Experiment II). These results indicate that the present variant of the word-superiority effect has a verbal locus (left hemisphere) rather than a visual-spatial locus (right hemisphere).
\end{abstract}

Letter strings typically are compared more rapidly and more accurately when one or both forms a familiar English word (e.g., Eichelman, 1970; Krueger, 1970b). Although words are readily encoded verbally, the speeded visual-comparison task, by its very nature, would seem to favor the use of visual-spatial rather than verbal information. Eichelman argued that words were indeed being compared on a physical or visual-spatial basis, because response time (RT) was shorter for same-case pairs (both rows in uppercase) than for different-case pairs (one row in upper case, the other in lowercase) for words as well as nonwords. If the words had been compared on a name or verbal basis, they ought not to have been faster in the same-case than in the different-case condition, because the comparison would have been at the same (i.e., name) level in both conditions.

The present study attempted to determine which process, verbal or visual-spatial, underlies or is the primary locus of the word superiority, by presenting words to either the left or the right visual hemifield. The test relied on the well-known relative specialization of the cerebral hemispheres: the left hemisphere for verbal material, the right hemisphere for spatial material (Gazzaniga, 1970; White, 1969). Items presented to the left hemifield are transmitted directly to the right hemisphere, and those presented to the right hemifield are transmitted directly to the left hemisphere. An enhanced word-superiority effect for the left (right) hemifield thus would be evidence for a visual-spatial (verbal) locus. Some previous visual-comparison studies have presented letter pairs or strings to the left and right hemifields. Left-right differences were not found in some cases (Cohen, 1973; Davis \& Schmit, 1971; Egeth, 1971), but other studies support the present position that name or verbal comparisons are relatively favored by the left hemisphere, and physical or visual-spatial

This study was supported bv NIH Grant HD04869 and HD09282. The author is grateful to Sheri Berenbaum, Jeffrey Wildfogel, Steve Silverman, and Richard Cohen for collecting and tabulating the data. Requests for reprints should be sent to Lester E. Krueger, Human Performance Center, Ohio State University, 404-B West 17th Avenue, Columbus, Ohio 43210. comparisons by the right hemisphere (Cohen, 1972; Gazzaniga, 1970, 1972; Geffen, Bradshaw, \& Nettleton, 1972; Gross, 1972).

\section{EXPERIMENTS I AND II}

The subject matched a letter presented in the center of the display with the other letters in the array, responding "yes" if any matched, "no" if none did. A four-letter string appeared to the left of the centered target letter on half of the trials and to the right on the other half. The four-letter string was a word on half of the trials and a nonword on the other half. It contained a letter which matched the target letter on half of the trials and did not on the other half.

The four-letter string was presented horizontally in Experiment I, vertically in Experiment II. The vertical arrangement was intended to eliminate several problems posed by the horizontal arrangement, such as the fact that the leftmost letter, which may be the most informative letter in a word (Bruner \& O'Dowd, 1958), is located closer to the fovea on right-hemifield than on left-hemifield displays (Dyer, 1973). Horizontal arrangement also may confound the factor of cerebral specialization or dominance with that of directional scanning. To eliminate the directional-scanning explanation, Bryden (1970) arrayed his items (four-letter first-order and fourth-order pseudowords presented unilaterally) both vertically and horizontally and found a right hemifield superiority on recognition in both cases. Barton, Goodglass, and Shai (1965) and Goodglass and Barton (1963), similarly, found more accurate identification of words in the right hemifield when vertical, unilateral arrays were presented.

\section{Method}

Apparatus. Stimuli were presented on a Gerbrands Model T-3B-1 tachistoscope at $4 \mathrm{~mL}$ intensity. Information on RT (measured in milliseconds by a specially built electronic timer) and on which telegraph key the subject pressed was punched on paper tape for subsequent computer analysis. To start each trial, the subject pressed a footpedal.

Stimulus materials. Each stimulus contained five uppercase letters (Prestype dry-transfer symbols, Futura Demibold, 36-point) on a $4 \times 6$ in. white index card. One (target) letter was 
centered on the card, with the other four appearing either to the left or to the right. The horizontal displays of Experiment I contained a 1.25-in. wide central region which remained unfilled (except for the target letter), and then, to both the left and right, four successive .375-in.-wide areas in each of which a letter might be centered (see Figure 1a). At 31 in., the horizontal visual angle for the nine letter locations was $8 \mathrm{deg}$. In the vertical displays of Experiment II, the .375-in.-high letters were each set at the bottom of an imaginary .5-in.-high box, with the second letter shifted .25 in. above the target letter and the third letter shifted .25 in. below (see Figure $1 \mathrm{~b}$ ). The nearest edge of the four-letter item was at least $1.1 \mathrm{deg}$ from the center of the fixated target letter. The small eccentricity was deemed sufficient, considering that the nasotemporal division of the retina in man is known to be extremely sharp and precise (Mitchell \& Blakemore, 1970; Trevarthen, 1970).

The four letters appeared on the left on half of the trials and on the right on the other half. Further, the center letter was present ("yes" response) among the four letters on half of the trials and was absent ("no" response) on the other half. The four letters were all different, and on half of the trials they formed a word. All words had A and AA ratings on the Thorndike and Lorge (1944) word count. Nonwords were formed by randomly permuting the letters in each letter position in the corresponding words for the left and right conditions.

The total set of 96 stimulus cards comprised three subsets of 32 cards which each represented all combinations of: (1) left-vs.-right location, (2) word vs. nonword, (3) target present vs. absent, and, (4) if present, whether the target matched the first, second, third, or fourth letter $(2 \times 2 \times 2 \times 4$ = 32). Each subset was randomized differently for each subject and appeared nearly equally as often as the first, second, or third subset. Except for the difference in horizontal vs. vertical arrangement, the displays were identical in Experiments I and II In Experiment $I$, the first subset of 32 cards presented was considered practice, but, in Experiment II, an additional 16 stimuli were prepared as a practice set.

Procedure. The subjects were instructed to look directly at the position of the center letter, as indicated by a prior fixation point, and to decide whether it was present in ("yes"), or absent from ("no"), the four-letter string to its left or right. Half of the subjects pressed the right key for "yes" and the left button for "no," and the other half had the reverse response assignment. Subjects were instructed to respond as quickly as possible but not at the expense of accuracy. A buzzer sounded briefly when the subject pressed the wrong key. All RTs which exceeded $3 \mathrm{sec}$, or on which the subject pressed the wrong key, were discarded.

On each trial, a fixation point appeared for $800 \mathrm{msec}$, then the stimulus card for $180 \mathrm{msec}$, and finally the fixation point for

\section{a. NTBS $\mathbf{N}$}

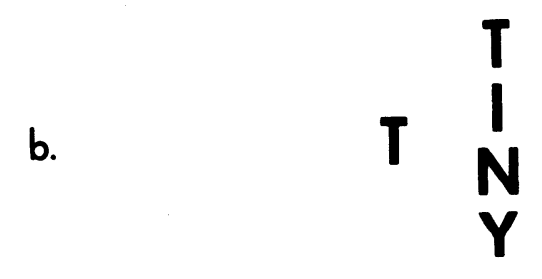

Figure 1. Examples of stimuli: (a) horizontal display (Experiment I) with a positive match on Postiion 1, left hemifield; (b) vertical display (Experiment II) with a positive match on Position 1, right hemifield.

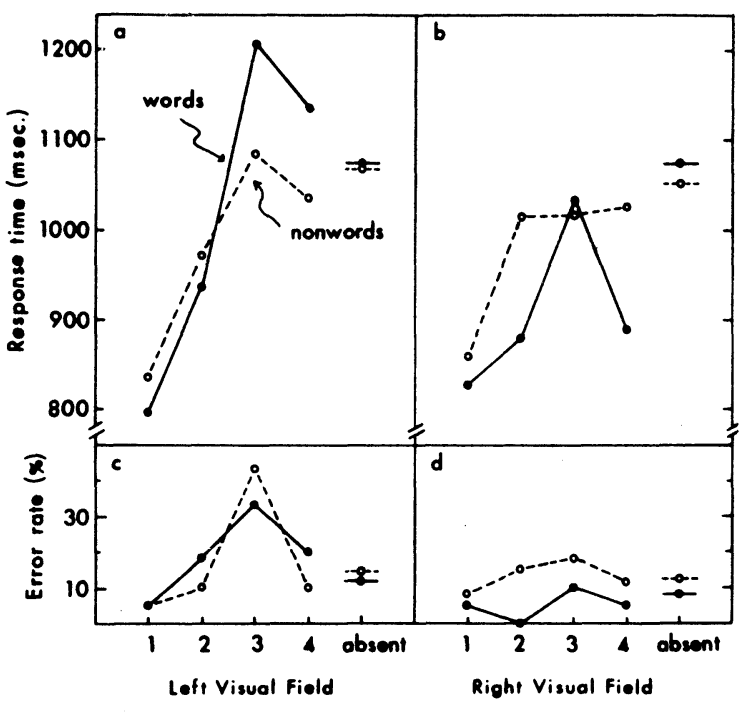

Figure 2. Experiment I: Mean RT and error rate, by serial position of target letter on positive trials, for horizontal, unilateral word and nonword displays.

another $3 \mathrm{sec}$. The $180-\mathrm{msec}$ exposure was chosen so as to keep errors within tolerable bounds, while not allowing the subject enough time to refixate to the left or right. The latency of a saccadic eye movement is typically about 180 to $250 \mathrm{msec}$. (Alpern, 1971; Saslow, 1967).

Subjects. Thirty City College of New York undergraduates, all native speakers of English, served as paid subjects in Experiment I, and a different group of 20 undergraduate, English-speaking, students served as subjects in Experiment II. All had 20/30 vision or better (corrected), as tested with a Snellen chart. All subjects in Experiment II were right-handed, as were all except four in Experiment $I$.

\section{Results}

All data were analyzed using the analysis of variance.

Experiment I: Horizontal displays. The results from Experiment I are shown in Figure 2. On RT, positive trials were significantly faster than negative ones $(p<.001)$, but no other main effect or interaction was significant. Error rate was significantly lower on words than on nonwords $(\mathrm{p}<.025)$ and on right hemifield items than on left hemifield ones $(p<.01)$. Because the Word-Nonword by Left-Right interaction was significant on error rate $(p<.01)$ and marginally significant on $R T$ $(p<.10)$, separate analyses were performed for words, nonwords, left hemifield, and right hemifield. Right hemifield words were responded to significantly faster $(p<.05)$ and more accurately $(p<.001)$ than left hemifield words, and the word-nonword difference on error rate was significant for the right-hemifield condition $(p<.001)$ but not for the left-hemifield condition.

Experiment II: Vertical displays. The results from Experiment II are shown in Figure 3. On RT, positive trials were significantly faster than negative ones, $\mathrm{p}<.001$, but no other main effect was significant. Error rate was lower overall for words than for nonwords, but 
only to a marginally significant extent, $p<.10$. The Left-Right by Word-Nonword interaction was significant, $p<.001$, reflecting the fact that a lower error rate for words was evident only for right-hemifield displays. As in Experiment I, separate analyses were performed for words, nonwords, left hemifield, and right hemifield. Among nonwords, there was a significantly greater error rate for right than for left hemifield, $\mathrm{p}<.025$; among words, the reverse effect, a greater error rate for left than for right hemifield, was not significant. The word-nonword difference on error rate was significant for the right-hemifield condition, $\mathrm{p}<.01$, but not for the left-hemifield condition.

\section{Discussion}

Experiments I and II are consistent in demonstrating a word-superiority effect only for the right hemifield, which projects directly to the language-dominant left hemisphere. The fact that the same effect was found for vertical displays as for horizontal displays would seem to rule out an alternative explanation of the results in terms of directional scanning.

It is striking that there was no word-superiority effect at all for the left-hemifield displays. The present task was a difficult one, involving a brief exposure of a peripherally located item, and thus might have favored a greater use of verbal processes (left hemisphere), as compensation for the lack of complete visual input. The task also might have favored the left hemisphere because it required that the four-letter items be searched letter by letter for the target character and thus be treated in analytical fashion, which may be the preferred mode of the left hemisphere (Nebes, 1974), rather than holistic fashion, which may be the preferred mode of the right hemisphere. Krueger (1970a), however, found the

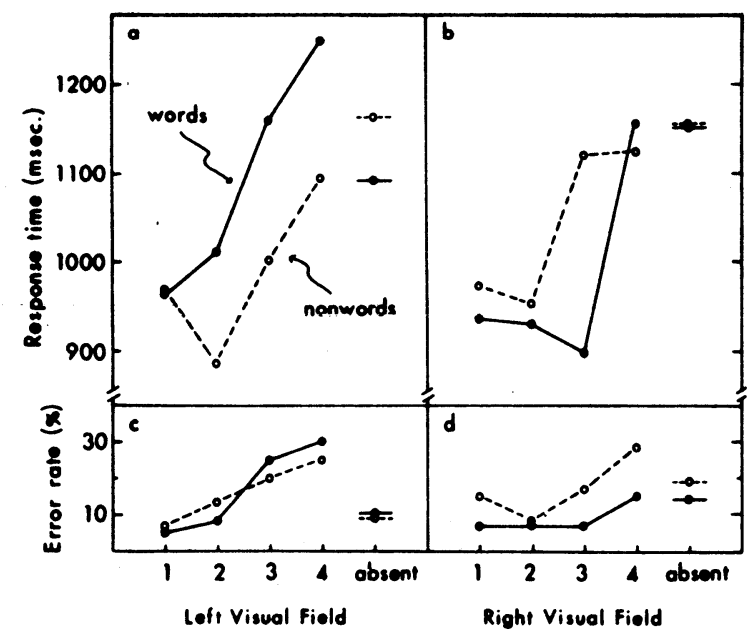

Figure 3. Experiment II: Mean RT and error rate, by serial position of target letter on positive trials, for vertical unilateral word and nonword displays. advantage for words over nonwords in a letter-search task to be located at the encoding stage, which presumably involves holistic processes, rather than at the comparison stage, in which the target letter is matched against the individual letters in the encoded item. Further, the fact that the nonwords differed not at all between the left and right hemifield conditions indicates that visual and verbal processes were well balanced in the task, allowing a fair opportunity for a visual locus, if any, to be revealed for the word-superiority effect. The present results therefore indicate that the word-superiority effect in letter detection has primarily a verbal locus. Whether the present conclusion holds for the word-superiority effect obtained with a different procedure, such as probe recognition or visual search, however, remains an open question. There may be not one familiarity or word-superiority effect, but several (cf. Krueger, in press).

\section{REFERENCES}

AlPern, M. Effector mechanisms in vision. In J. A. Kling \& L. A. Riggs (Ed.) Woodworth and Schlosberg's Experimental Psychology (3rd ed.). New York: Holt, Rinehart, \& Winston, 1971. Pp. 369-394.

Barton, M. I., Goodglass, H., \& Shai, A. Differential recognition of tachistoscopically presented English and Hebrew words in right and left visual fields. Perceptual and Motor Skills, $1965,21,431-437$.

Bruner, J. S., \& O' Dowd, D. A note on the informativeness of parts of words. Language and Speech, 1958, 1, 98-101.

Bryden, M. P. Left-right differences in tachistoscopic recognition as a function of familiarity and pattern orientation. Journal of Experimental Psychology, 1970, 84, 120-122.

Conen, G. Hemispheric differences in a letter classification task. Perception \& Psychophysics, 1972, 11, 139-142.

Conen, G. Hemispheric differences in serial versus parallel processing. Journal of Experimental Psychology, 1973, 97, 349-356.

Davis, R., \& Schmit, V. Timing the transfer of information between hemispheres in man. Acta Psychologica, 1971, 35, 335-346.

DYER, F. N. Interference and facilitation for color naming with separate bilateral presentations of the word and color. Journal of Experimental Psychology, 1973, 99, 314-317.

Egetr, H. Laterality effects in perceptual matching. Perception \& Psychophysics, 1971, 9, 375-376.

Eichelman, W. H. Familiarity effects in the simultaneous matching task. Journal of Experimental Psychology, 1970, 86, 275-282.

Gazzaniga, M. S. The bisected brain. New York: Appleton-Century-Crofts, 1970.

Gazzaniga, M. S. One brain-two minds? American Scientist, 1972, 60, 311-317.

Geffen, G., Bradshaw, J. L., \& Netrleton, N. C. Hemispheric asymmetry: Verbal and spatial encoding of visual stimuli. Journal of Experimental Psychology, 1972, 95, 25-31.

Goodglass, H., \& Barton, M. Handedness and differential perception of verbal stimuli in left and right visual fields. Perceptual and Motor Skills, 1963, 17, 851-854.

Gross, M. M. Hemispheric specialization for processing of visually presented verbal and spatial stimuli. Perception \& Psychophysics, 1972, 12, 357-363.

Krueger, L. E. Search time in a redundant visual display. Journal of Experimental Psychology, 1970, 83, 391-399. (a) 
KrUeger, L. E. Visual comparison in a redundant display. Cognitive Psychology, 1970, 1, 341-357. (b)

KRUEGER, L. E. Familiarity effects in visual information processing. Psychological Bulletin. 1975, in press.

Mitchell, D. E., \& Blakemore, C. Binocular depth perception and the corpus callosum. Vision Research, 1970, 10, 43-47.

NEBES, R. D. Hemispheric specialization in commissurotomized man. Psychological Bulletin, 1974, 81, 1-14.

SAsLow, M. G. Latency for saccadic eye movement. Journal of the Optical Society of America, 1967, 57, 1030-1036.
ThORNDIKE, E. L., \& LORGE, I. The teacher's word book of 30.000 words. New York: Teachers College, Columbia University, Bureau of Publications, 1944.

Trevarthen, C. Experimental evidence for a brainstem contribution to visual perception in man. Brain. Behaviour and Evolution, 1970, 3, 338-352.

WhITE, M. J. Laterality differences in perception: A review. Psychological Bulletin, 1969, 72, 387-405.

(Received for publication July 10, 1975.) 